\title{
EDITORIAL
}

\section{Multifaces of Modern Analytical Chemistry}

\author{
Érico M. M. Flores (iD $ه$ \\ Full Professor \\ Departamento de Química, Universidade Federal de Santa Maria \\ Santa Maria, RS, Brazil
}

The Brazilian Journal of Analytical Chemistry has completed ten years with focus on the integration between academia, research centers, and industry. In this regard, the BrJAC showed rapid growth and great acceptance by the analytical chemistry community and other related science disciplines. There was a rapid increase in its impact factor and visibility, showing that the BrJAC arrived at a good time for analytical chemistry, which is currently one of the most vigorous fields, with researchers cited among the top scientists in the world. Currently, new materials, information technology, advances in instrumentation and the interaction of other areas with analytical chemistry have brought many advances and new developments. In this sense, important advances are observed in areas related to environmental control, the use of chemometrics, quality control of food and pharmaceutical products, among others.

It is possible to observe a clear trend towards the development of faster and less invasive methods, with better detectability and selectivity. Another important aspect that is becoming increasingly more present in analytical chemistry papers refers to the use of methods in agreement to the principles of green chemistry, which presupposes, among other aspects, the use of lower volumes of concentrated reagents and, obviously, the generation of less laboratory waste and a lower need for waste treatment for disposal or eventual reuse.

In this issue of the BrJAC, most of these developments are covered. We can read the excellent Interview with a very famous Chilean researcher who has developed plenty of important analytical methods, being the pioneer of a clever system based on a microextraction system using a rotating-disk sorptive extraction technique that has many important applications.

The BrJAC also provides a look at a current challenge related to arsenic speciation. It is well known that arsenic speciation methods are important to understand the toxicity or essentiality of its species for environmental and biological studies. Here, a Point of View is presented from an expert in this field, showing the possibilities and recent trends. Sample preparation methods, such as the use of solid phase extraction systems, have been applied to many matrices, even enabling the on-site separation of As species.

The importance of the study of polymorphism and analytical techniques for the structural characterization of food products with a special focus on chocolate is highlighted in the Letter section. Solid state characterization is fundamental for the food Industry. It allows the development of food products with many advantages, such as better formulations, processability, stability and functionality.

The Review section provides a deep and critical revision of chemometrics applications, with focus on the quality by design approach for the development of new analytical methods. The authors are experts in this field and also demonstrated the effect of the quality by design approach on the recent changes in traditional manufacturing, helping to save both energy and time. It is possible to see that there is a very good expectation for new applications in many areas in addition to the already well-established use for pharmaceutical purposes.

Cite: Flores, E. M. M. Multifaces of Modern Analytical Chemistry. Braz. J. Anal. Chem., 2021, 8 (30), pp 1-2. doi: http://dx.doi. org/10.30744/brjac.2179-3425.editorial.emmflores.N30 
In this issue, two interesting Articles are included. The first describes a comprehensive study related to the effect of ultraviolet radiation (UV-C) on many kinds of paper. The authors performed a full characterization of UV-C irradiated samples with a multitude of techniques (SEM-EDS, DTC-TGA, FTIR, XRD among others). The second article shows the complete evaluation of a validated method using RPHPLC that provided the accurate determination of pharmaceutical products. A full degradation study was also described, showing the suitability for purity assessment in analytical laboratories.

Finally, the BrJAC also presents a Technical Note with a relatively recent improvement in the field of atomic absorption spectrometry (HR-CS FAAS): an important application of HR-CS FAAS for the analysis of B7-diesel oil and multi-element determination of trace elements. The authors used a smart strategy based on the formation of microemulsions that allowed a fast and accurate analysis.

Therefore, this issue brings many exciting articles of researchers from many countries showing that the BrJAC is becoming more important to the field of analytical chemistry and that authors are increasingly more often considering this journal as a suitable channel to spread their research at a high level.

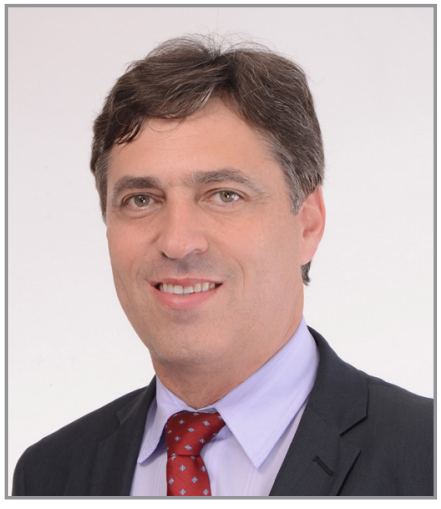

Érico Marlon de Moraes Flores is currently Full Professor of the Department of Chemistry at the Federal University of Santa Maria, Santa Maria, RS, Brazil. He works mainly in research and technological development involving atomic spectrometry and the use of alternative energies, such as ultrasound and microwave for sample preparation with application in several laboratories and also for the intensification of industrial processes. He has also worked in the field of quality control of pharmaceutical products, food, nanomaterials and in the extraction and analytical developments for the determination of rare earth elements.

CV: http://lattes.cnpq.br/7167629055579212

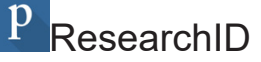

